\title{
Multiphase Resonant Converter with Output Current Multiplier for Battery Charger Applications
}

\author{
C. Brañas ${ }^{1}$, J.C. Viera², F.J. Azcondo ${ }^{1}$, R. Casanueva ${ }^{1}$, D. Anseán ${ }^{2}$ \\ ${ }^{1}$ University of Cantabria \\ Electronics Technology, Systems and Automation \\ Engineering Department \\ Ave. de los Castros s/n 39005 Santander, SPAIN \\ branasc@unican.es \\ ${ }^{2}$ University of Oviedo \\ Department of Electrical Engineering \\ Campus de Gijón, Módulo 3. \\ 33204 Gijón, SPAIN \\ viera@uniovi.es
}

\begin{abstract}
This paper presents the analysis and design of a multiphase resonant converter suitable for high-current lowvoltage battery charger applications. In order to reduce the conduction losses, the inverter stage of the converter is obtained from the parallel connection of $N$ class $\mathrm{D} L C_{p}$ resonant inverters. In the same way, the output stage is based on a current multiplier, obtained from the parallel connection of two current doubler rectifiers. The regulation of the charging current is implemented at constant frequency, modifying the phase displacement of the drive signals. The battery charger is designed for highperformance Absorbent Glass Mat (AGM) battery; at present, widely used in micro-hybrid vehicles.
\end{abstract}

\section{INTRODUCTION}

This paper proposes a multiphase resonant converter with a new current multiplier as output stage for lead acid battery charger applications. Currently, the lead acid battery technology is being upgraded to fulfil the power demanded for microhybrid cars at lower cost than others advanced technologies [1]. The high-performance AGM battery is an example of this development. These batteries present lower internal resistance for additional power, deeper discharges and quicker recharging, higher corrosion resistance for longer service life with reduced self-discharge when stored on the shelf.

Chargers for low-voltage high-current batteries should provide high output current in order to comply with the charging profile of the battery. In high current applications, the conduction losses are a major design issue [2]. Nowadays, the new wide bandgap devices (WBD) are making easier to achieve high efficiency at high current levels with important simplifications of the power circuit [3]. However, the cost of WBD is still high. As alternative, multiphase structures divide the current among different parallelized stages, which enables to use low cost components [4].

\section{CHARGING METHOD}

The main features of the AGM battery, specially designed for vehicles with advanced Start-Stop functionality with regenerative braking are: $12 \mathrm{~V}$ nominal voltage, $105 \mathrm{Ah}$ nominal capacity at $\mathrm{C} / 20$ measured at $25^{\circ} \mathrm{C}$ and low internal resistance. Conventional constant current - constant voltage (CC-CV) based method is shown in Fig. 1 using three experimental profiles, with a constant current stage of 10A, 15A and 20A respectivily.

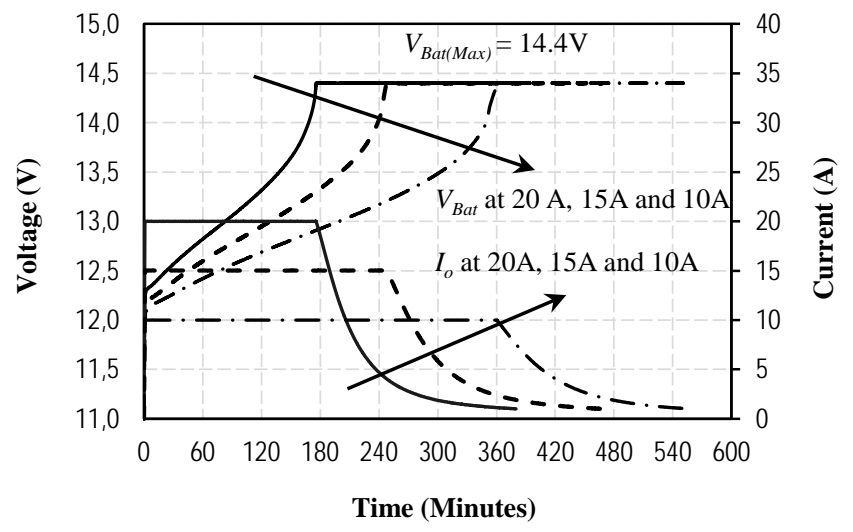

Fig. 1. Experimental charging profiles at 10 A, 15 A and 20 A for an AGM battery. (Varta Silver Dynamic AGM).

Voltage and current profiles shown in Fig. 1 were obtained using the High Power Equipment SBT 10050. From Fig. 1, it is observed that the constant-current stage is the most efficient for charging a fully discharged battery. During this stage, the battery accepts the highest current level. The experimental study of the battery under test determines that the maximum battery voltage must be limited at $V_{\text {Bat(Max) }}=14.4 \mathrm{~V}$ in order to avoid electrolyte loss. In this way, during the constant-voltage stage, the voltage is set to the maximum, $V_{\text {Bat(Max) }}=14.4 \mathrm{~V}$, so that the battery current decreases as the charging process continues. Finally, it should be taken into account that in order to avoid an overcharge situation, gas production and consequently the battery degradation, limitation of the current ripple is mandatory.

\section{MULTIPHASE $L C_{P}$ RESONANT CONVERTER}

The proposed battery charger is based on a multiphase resonant converter. The AC side is a multiphase resonant inverter, which consists of $N$ paralleled $L C_{p}$ class D sections [5]. The DC side consists of a new current multiplier stage, derived from the current-doubler rectifier [6]. The circuit and the battery in steady-state with its internal impedance, $r_{B a t}$, plus an ideal voltage source $V_{\text {Bat }}$, are represented in Fig.2. 


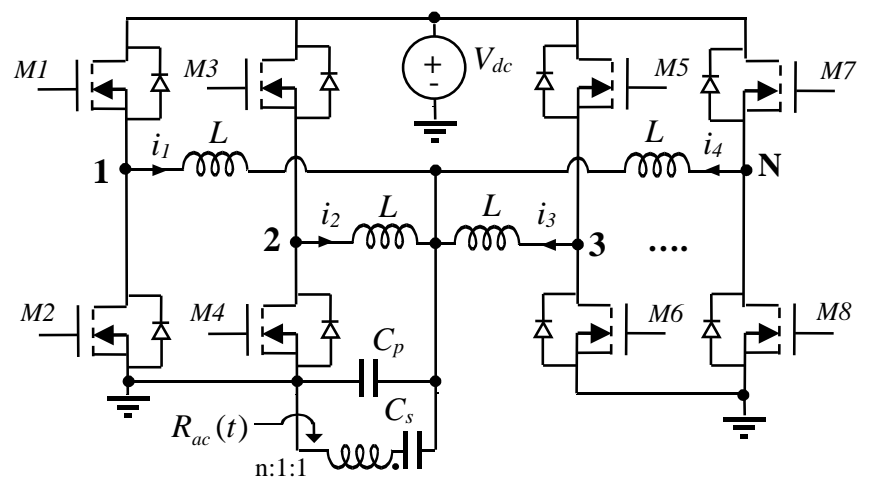

of two current-doubler rectifiers. The advantages of the proposed configuration are similar to the original currentdoubler rectifier and are related to lower conduction losses and simplification of the transformer in comparison to its center tap rectifier counterpart [7]. Since the output filter removes the high frequency ripple, the low ripple approximation [8] is used for modeling in steady state the proposed rectifier stage. The current path for positive and negative semi cycles of the AC current are shown in Fig. 4.

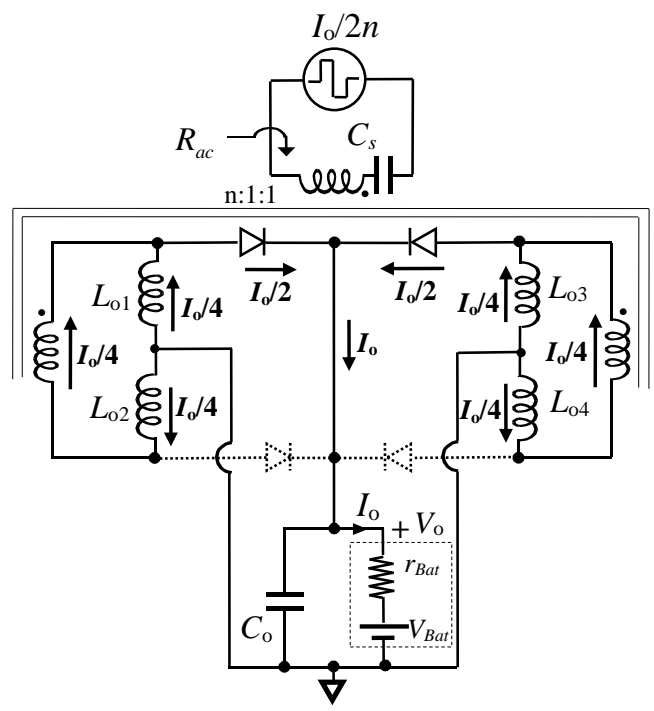

The proposed converter is analyzed considering the phase angle of all midpoint voltages $v_{k}$ independents. To illustrate this assumption, the midpoint voltages $v_{k}$ are shown in Fig.3.

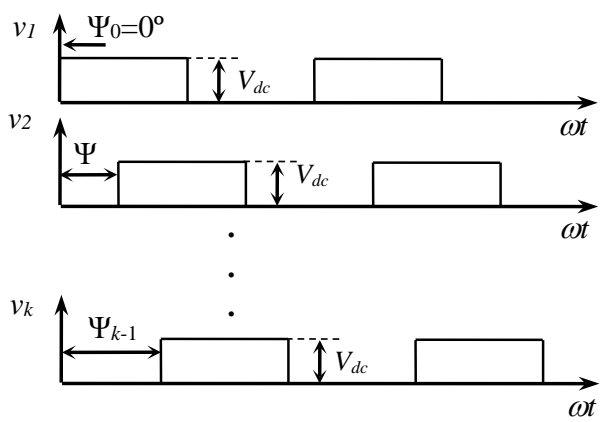

Fig.3. Multiphase controlled $L C_{p}$ resonant inverter. Output voltages of each inverter section.

Using the fundamental approximation, the input voltages, $v_{k}$, can be represented by phasors given in (1),

$$
\mathbf{V}_{\mathbf{k}}=\frac{2 V_{d c}}{\pi} \cdot e^{-j \Psi_{k-1}}
$$

where $k \in[1,2 \ldots N]$ and $N$ being the number of phases of the resonant inverter stage.

\section{A. Current Multiplier}

The charging current circulating through the output rectifier stage is a major design issue. A current multiplier is proposed as rectifier stage, which is obtained from the parallel connection

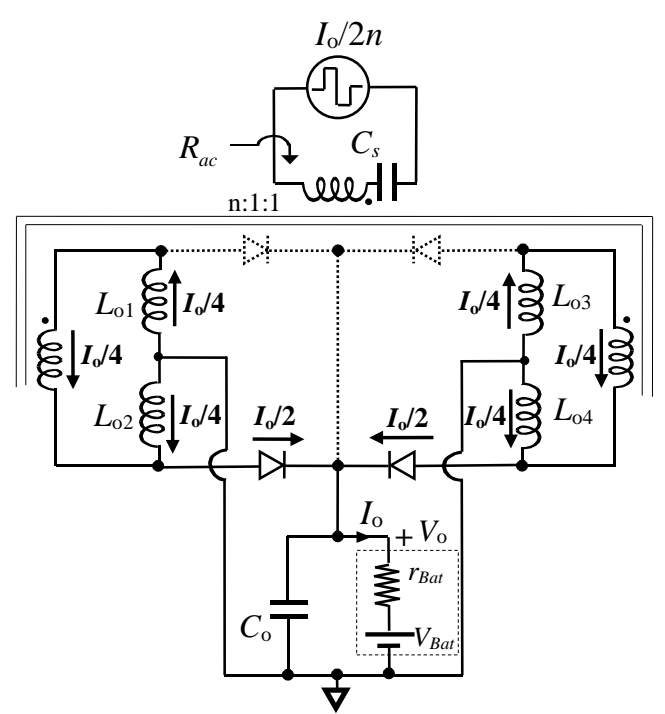

Fig. 4.Proposed output current multiplier. Current path for positive and negative cycles of the AC current.

With the first harmonic of a square waveform, the relationship between the AC and DC side currents is given by,

$$
I_{o}=\frac{n \pi}{2} \cdot \hat{I}_{a c}
$$

where $\hat{I}_{a c}$, is the amplitude of the transformer primary current and $n$ is the transformer turns ratio $(n: 1: 1)$. On the other hand, the output voltage, $V_{o}$, is obtained as the mean value of the full wave rectified voltage. 


$$
V_{o}=\frac{2}{n \pi} \cdot \hat{V}_{a c}=r_{\text {Bat }} \cdot I_{o}+V_{\text {Bat }}
$$

From (2) and (3), the battery model is represented in the AC side by,

$$
\hat{V}_{a c}=\frac{n^{2} \pi^{2}}{4} r_{\text {Bat }} \cdot \hat{I}_{a c}+\frac{n \pi}{2} \cdot V_{B a t},
$$

From (4), the rectifier stage is reflected to the AC side as the equivalent resistance given in (5),

$$
R_{a c}=\frac{\pi^{2}}{4} n^{2} R_{o}=\frac{\pi^{2}}{4} n^{2}\left(r_{B a t}+\frac{V_{B a t}}{I_{o}}\right)
$$

The value of $r_{B a t}$ depends on the battery state of charge (SOC) and for AGM technology is rather small $\left(r_{B a t}<<1 \Omega\right)$. For simplicity sake, $r_{B a t}$ is neglected and only the term $V_{B a t} / I_{O}$ is considered in (5). Finally, by using (1-5), the simplified circuit of the resonant converter is depicted in Fig. 5.

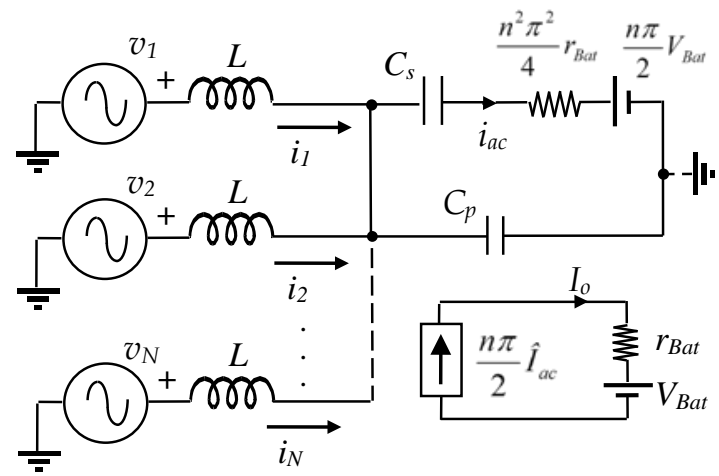

Fig. 5. Simplified circuit by using the fundamental approximation for circuit analysis purposes.

The analysis of the proposed resonant converter is carried out by using the simplified circuit shown in Fig.5. The main features of the resonant converter are defined by parameters shown in Table I.

TABLE I.

PARAMETERS OF THE MULTIPHASE $L C_{p}$ INVERTER

\begin{tabular}{|c|c|c|}
\hline $\begin{array}{c}\text { Parallel } \\
\text { Resonant } \\
\text { Frequency }\end{array}$ & $\begin{array}{c}\text { Parallel } \\
\text { Characteristic } \\
\text { Impedance }\end{array}$ & $\begin{array}{c}\text { Parallel } \\
\text { Quality } \\
\text { Factor }\end{array}$ \\
\hline$\omega_{p}=\frac{1}{\sqrt{L C_{p} / N}}$ & $Z_{p}=\omega_{p} L=\frac{N}{\omega_{p} C_{p}}$ & $Q_{p}=\frac{N R_{a c}}{Z_{p}}$ \\
\hline
\end{tabular}

\section{B. Distribution of Currents}

The proposed $L C_{p}$ resonant converter is designed as a current source by fixing the switching frequency at $\omega=\omega_{p}$, where $\omega_{p}$ is the parallel resonant frequency, given in Table I. During the constant current stage, the current source behavior of the converter provides inherent current limitation protecting the battery and extending its life. The analysis of the resonant converter is carried out considering the switching frequency, $\omega=\omega_{p}$, constant and the angles $\Psi_{0}, \Psi_{1}, \Psi_{N-1}$ as the control parameters. The inductors' current phasors are given in (6),
$\mathbf{I}_{\mathbf{k}}=\frac{2 V_{d c}}{\pi Z_{p}} \times\left\{\frac{Q_{p}}{N} \sum_{m=1}^{N} \cos \Psi_{m-1}-\sin \Psi_{k-1}-j\left[\cos \Psi_{k-1}+\frac{Q_{p}}{N} \sum_{m=1}^{N} \sin \Psi_{m-1}\right]\right\}$

The phasor of the output current in the AC side, $\mathbf{I}_{\mathbf{a c}}$, is calculated by (7).

$$
\mathbf{I}_{\mathrm{ac}}=\frac{2 V_{d c}}{\pi Z_{p}}\left\{\sum_{m=1}^{N} \sin \Psi_{m-1}+j \sum_{m=1}^{N} \cos \Psi_{m-1}\right\}
$$

From (7) it can be verified the current source behavior as the output current in the AC side has no dependence on the load. The optimum operation of the multiphase converter is achieved when the distribution of currents among the inverter sections is balanced. Under this condition, each inverter section handle only a fraction of the AC side load current, so that $\hat{I}_{k} \approx \hat{I}_{a c} / N$. For maximum output current $\left(\Psi=0^{\circ}\right)$, the ratio of the AC output current, $\hat{I}_{a c}$, to the inductors current amplitude, $\hat{I}_{k}$, is given by,

$$
\frac{\hat{I}_{a c}}{\hat{I}_{k}}=\frac{N}{\sqrt{1+Q_{p}^{2}}}
$$

In order to achieve a balanced current sharing among all resonant phases, it is desirable a value of quality factor, $Q_{p}<1$.

\section{Variation of the Quality Factor}

During the charging process, $R_{a c}$ changes according to $V_{B a t}$ and $I_{o}$. The relationship between $V_{B a t}$ and $I_{O}$ is given by the charging profile of the battery, shown in Fig. 1. During the constant current stage, $R_{o}$ rises up to the value $V_{\text {Bat(Max) }} / I_{o}$. At the end of the constant current stage, the output power is maximum. Working with (2) and (7), the maximum charging current is achieved at $\Psi_{0}=\Psi_{1}=\Psi_{N-1}=0^{\circ}$ and is given by,

$$
I_{o}=\frac{n V_{d c}}{Z_{p}} \cdot N
$$

From (9) it can be observed that the output current capability of the multiphase converter can be enhanced by increasing the number, $N$, of paralleled phases. On the other hand, during the constant voltage stage, the charging current must be adjusted in order to avoid the battery voltage exceeds the maximum value,

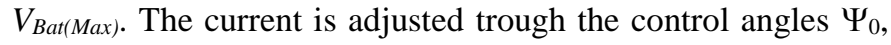
$\Psi_{1}, \Psi_{N-1}$, while keeping constant the switching frequency.

Different patterns for adjusting $\Psi_{1}, \Psi_{2}$ and $\Psi_{N-1}$ are possible. For any value of $N$, the full control of the charging current is achieved if the phase shift is evenly distributed among all phases, e.g. $\Psi_{0}=0^{\circ}, \Psi_{1}=\Psi, \Psi_{2}=2 \Psi \ldots \Psi_{N-1}=N \Psi$. In this case, the minimum current $I_{o}=0 \mathrm{~A}$ is achieved at $\Psi=360^{\circ} / N$. This pattern requires of $N$ independent control signals.

For even values of $N$, the full control of the charging current can be achieved by shifting the phases by pairs, e.g. for $N=4$ then $\Psi_{0}=\Psi_{1}=0^{\circ}$ and $\Psi_{2}=\Psi_{3}=\Psi$. In this case, the minimum $I_{o}=0 \mathrm{~A}$ is achieved at $\Psi=180^{\circ}$ and only two independent control signals are required which implies a simplification of the control circuit. The normalized amplitude of the charging current, $I_{o}$, is depicted in Fig. 6 as a function of the control angle, $\Psi$ 
considering the control pattern where all phases are evenly shifted.

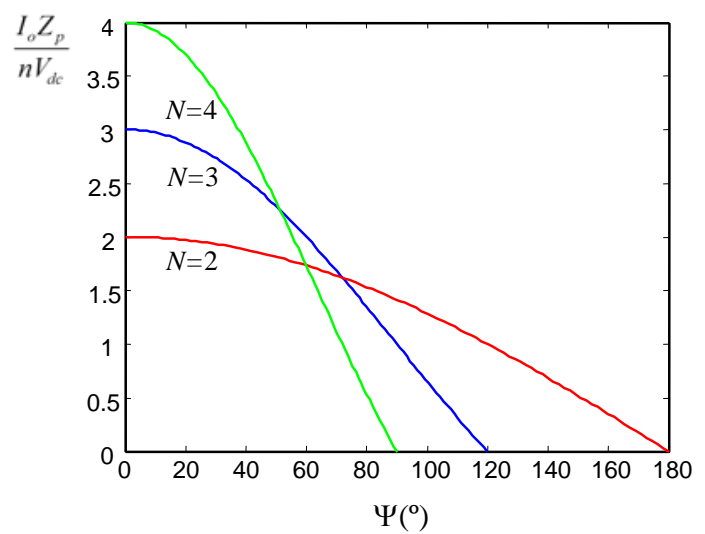

Fig. 6. Amplitude of the normalized charging current, $I_{o}$, as a function of the control angle, $\Psi$, for $N=2,3$ and 4 . All phases are evenly shifted, $\Psi_{0}=0^{\circ}, \Psi_{1}=\Psi, \Psi_{2}=2 \Psi \ldots \Psi_{N-1}=N \Psi$.

The reduction of the charging current during the constant voltage stage leads to a significant increment of the equivalent resistance $R_{o}$ and consequently, the reflected impedance in the AC side, $R_{a c}$, and the quality factor $Q_{p}$ also increase. Assuming that $V_{\text {Bat(Max) }}$ is constant and working with (7), (5) and (2), the variation of the quality factor is given in (10),

$$
Q_{p}=\frac{\pi^{2} n V_{\text {Bat }(\operatorname{Max})} N}{2 V_{d c} \sqrt{\left(\sum_{m=1}^{N} \sin \Psi_{m-1}\right)+\left(\sum_{m=1}^{N} \cos \Psi_{m-1}\right)}}
$$

The variation of the quality factor due to the current adjustment is represented in Fig. 7 as a function of the control angle $\Psi$.

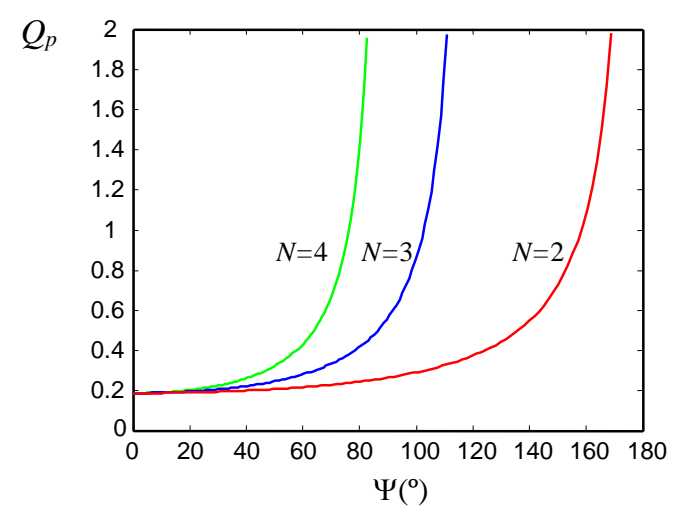

Fig. 7. Quality factor variation during charging at constant voltage. The parameters are: $n=2 ; N=2,3$ and $4 ; V_{B a t(\text { Max })}=14.4 \mathrm{~V}$ and $V_{d c}=400 \mathrm{~V}$. All phases are evenly shifted, $\Psi_{0}=0^{\circ}, \Psi_{1}=\Psi, \Psi_{2}=2 \Psi \ldots \Psi_{N-1}=N \Psi$.

\section{EFFICIENCY OF THE CONVERTER}

The overall efficiency of the resonant converter is calculated by,

$$
\eta=\eta_{I} \eta_{R},
$$

where $\eta_{I}$ is the efficiency of the resonant inverter stage and $\eta_{R}$ is the efficiency of the output rectifier stage.

\section{A. Efficiency of the Inverter.}

The conduction losses are significant in high current applications. The efficiency of the resonant inverter stage, $\eta_{I}$, considering the conduction losses only [9] is

$$
\eta_{I}=\frac{1}{1+\frac{r}{R_{a c}} \cdot \frac{\sum_{k=1}^{N}\left\{\left[\frac{Q_{p}}{N} \sum_{m=1}^{N} \cos \Psi_{m-1}-\sin \Psi_{k-1}\right]^{2}+\left[\cos \Psi_{k-1}+\frac{Q_{p}}{N} \sum_{m=1}^{N} \sin \Psi_{m-1}\right]^{2}\right\}}{\left[\sum_{m=1}^{N} \sin \Psi_{m-1}\right]^{2}+\left[\sum_{m=1}^{N} \cos \Psi_{m-1}\right]^{2}}}
$$

The resistor $r$ in (12) represents the $r d s_{\text {on }}$ of the transistors as well as the ESR of the inductors

$$
r=r d s_{o n}+\frac{Z_{L}}{Q_{L}},
$$

where $Z_{L}$ is the impedance of the inductor at the switching frequency and $Q_{L}$ is the quality factor of the inductor. The efficiency, $\eta_{I}$, is plotted in Fig. 8 as a function of the control angle, $\Psi$. For all cases, the output current is the same, the value of $r=1 \Omega$ is assumed and the transformer turns ratio is $n=2$. The value of $Z_{p}=80 \Omega$ is considered for $N=4$ and values of $Z_{p}=60 \Omega$ and $40 \Omega$ for $N=3$ and 2 , the respectively. The plot was obtained taking into account the variation of $Q_{p}$ given in (10) and a phase shift evenly distributed among all phases.

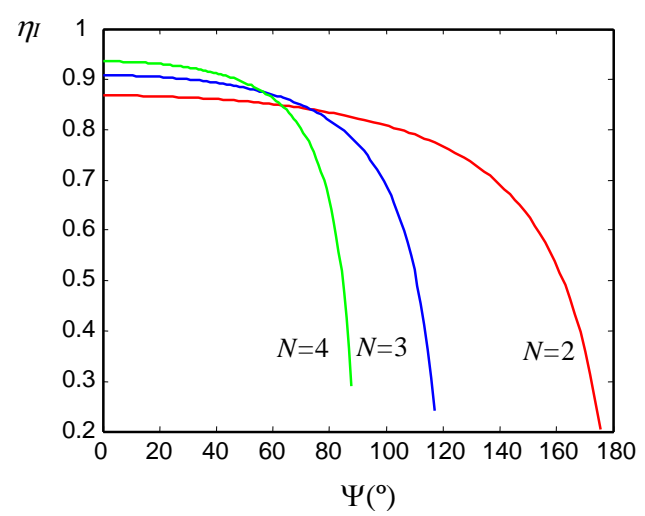

Fig. 8. Efficiency of the resonant inverter stage, $\eta_{I}$, as a function of the control angle, $\Psi$, for $N=2,3$ and $4, r=1 \Omega$ and $n=2$. All phases are evenly shifted, $\Psi_{0}=0^{\circ}, \Psi_{1}=\Psi, \Psi_{2}=2 \Psi \ldots \Psi_{N-1}=N \Psi$.

From Fig. 8, it is observed that the highest value of efficiency,

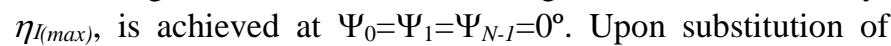
$\Psi_{0}=\Psi_{1}=\Psi_{N-1}=0^{\circ}$ in (12), the maximum value of efficiency is obtained, which is a function of the ratio $r / R_{a c}$, the initial value of the quality factor, $Q_{p}$, and the number of phases, $N$.

$$
\eta_{I(\max )}=\frac{1}{1+\frac{r}{N R_{a c}} \cdot\left(1+Q_{p}^{2}\right)}=\frac{1}{1+\frac{r}{Z_{p}} \cdot\left(\frac{1+Q_{p}^{2}}{Q_{p}}\right)}
$$

From (14), the maximum efficiency of the inverter stage, $\eta_{\text {I(max) }}$, can be improved by increasing the number of phases $N$ as it is also observed in Fig. 5. 
The value of $\eta_{I_{(\max )}}$ is used as criterion in order to define the appropriate number of phases, $N$. From (14), it is also considered that $\eta_{I(\max )}$ can be improved by increasing $R_{a c}$. The straightforward way for increasing $R_{a c}$ is by the transformer turns ratio, $n$. However, it should be considered that $Q_{p}$ also increases, which could jeopardize the ZVS mode of the converter transistors. Taking into account the tight correlation among, $N, Q_{p}, n$ and $r / R_{a c}$, the design process to find a suitable value of such parameters may involve iterative cycles.

\section{B. Efficiency of the Output Current Multiplier.}

The proposed two-phase output current multiplier presents lower conduction losses than its center tap rectifier and the conventional current-doubler counterparts. The rectifier efficiency, considering only the conduction losses, is obtained from the analysis of the current paths shown in Fig. 4. Considering a lossless transformer, the efficiency is calculated according to,

$$
\eta_{R}=\frac{1}{1+\frac{V_{D}+\left(\frac{r_{D}}{2}+\frac{r_{L F}}{4}\right) I_{o}}{V_{\text {Bat(Max })}}},
$$

where $V_{D}$ and $r_{D}$ are the voltage and dynamic resistance of the linear model of the diode and $r_{L F}$ is the ESR of the filter inductors $L_{01}-L_{04}$. In comparison to the resonant inverter stage, where the maximum efficiency is achieved at full load operation, the efficiency of the rectifier stage improves under light load condition. This characteristic yields an overall efficiency of the resonant converter with a wide flat zone.

\section{SWITCHING MODE AND COMPLEX POWER}

The switching losses are minimized by achieving the zerovoltage switch (ZVS) in the primary side of the converter. The ZVS mode requires a phase delay of the resonant current with respect to the input voltage. In order to determine the power factor angle, $\phi_{k}$, of each transistors leg and study the power distribution, the complex power for each generator $v_{k}$ is calculated in (16). From (16) the power factor angle, $\phi_{k}$ is obtained by using $\phi_{k}=\operatorname{angle}\left(\boldsymbol{S}_{k}\right)$ as a function of the control angles, $\Psi_{0}, \Psi_{1}, \Psi_{N-1}$, the number of phases, $N$, and the quality factor, $Q_{p}$.

$$
\begin{aligned}
& \mathbf{S}_{\mathbf{k}}=\frac{2 V_{d c}^{2}}{\pi^{2} Z_{p}} \times \\
& \left\{\begin{array}{l}
\frac{Q_{p}}{N}\left[\cos \Psi_{k-1} \cdot \sum_{m=1}^{N} \cos \Psi_{m-1}+\sin \Psi_{k-1} \cdot \sum_{m=1}^{N} \sin \Psi_{m-1}\right] \\
+j\left[1+\frac{Q_{p}}{N}\left(\cos \Psi_{k-1} \cdot \sum_{m=1}^{N} \sin \Psi_{m-1}-\sin \Psi_{k-1} \cdot \sum_{m=1}^{N} \cos \Psi_{m-1}\right)\right]
\end{array}\right.
\end{aligned}
$$

Upon substitution $\Psi_{0}=\Psi_{1}=\Psi_{N-1}=0^{\circ}$ in (16), the power factor angle at maximum output current is obtained:

$$
\phi_{1,2 \ldots N}=\arctan \left(\frac{1}{Q_{p}}\right),
$$

The value of the quality factor defines the ratio active power to reactive power handled by each inverter section. In this way, a high value of $Q_{p}$ reduces the reactive energy in the resonant converter and improves the efficiency. However, some amount of reactive energy must be accepted for assuring the ZVS mode of all transistors. The minimum value of power factor angle $\phi_{z v s}$ depends on the dead time, $t_{d}$, of the driver transistors and the switching frequency [10].

$$
\phi_{z v s}=\frac{t_{d}}{T_{s}} \cdot 360^{\circ}
$$

The total complex power, $\mathbf{S}=\mathbf{S}_{\mathbf{1}}+\mathbf{S}_{2} \ldots+\mathbf{S}_{\mathbf{N}}$, is calculated by,

$$
\begin{aligned}
& \mathbf{S}=P+j Q= \\
& \frac{2 V_{d c}^{2} Q_{p}}{\pi^{2} Z_{p} N} \cdot\left\{\left[\sum_{m=1}^{N} \cos \Psi_{m-1}\right]^{2}+\left[\sum_{m=1}^{N} \sin \Psi_{m-1}\right]^{2}\right\}+j \frac{2 N V_{d c}^{2}}{\pi^{2} Z_{p}}
\end{aligned}
$$

\section{DESIGN OF THE RESONANT CONVERTER}

Following, the step-by-step design procedure is summarized.

1) The maximum battery voltage is $V_{\text {Bat(Max) }}=14.4 \mathrm{~V}$. Although the maximum charging current specified in the charging profile is $20 \mathrm{~A}$, the output current capability of the circuit is set at $I_{o}=25 \mathrm{~A}$. The converter supply voltage is $V_{d c}=400 \mathrm{~V}$ which is the output voltage of a previous front-end PFC stage. The switching frequency is set at $\omega_{p}=2 \pi(125 \mathrm{kHz})$.

2) The transformer turns ratio, $n$, and the number of phases, $N$, are calculated taking into account as criterion the maximum efficiency of the inverter stage, $\eta_{I(\operatorname{Max}) \text {. Upon }}$ substitution of (9) and (10) into (14), $\eta_{\text {I(Max) }}$ as a function of the converter parameters is obtained,

$$
\eta_{I(\max )}=\frac{1}{1+\frac{\pi^{2} r I_{o} V_{B a t(M a x)}}{2 N V_{d c}^{2}}+\frac{2 r I_{o}}{n^{2} \pi^{2} N V_{B a t(\operatorname{Max})}}} \approx \frac{1}{1+\frac{2 r I_{o}}{n^{2} \pi^{2} N V_{B a t(\operatorname{Max})}}}
$$

The transistors are low cost IRF840LC with $r_{d s(o n)}=0.83 \Omega$. Considering the ESR of the resonant inductors, a worst case $r=2 \Omega$ is assumed. Upon substitution in (20), the pair $n=2$ and $N=4$ yields $\eta_{\text {I(Max) }}=0.957$, high enough to achieve an overall efficiency above $\eta=0.8$.

3) The efficiency of the rectifier stage is calculated taking into account the conduction loss model of the Schottky diode STPS60SM200CW from ST with $V_{D}=0.58 \mathrm{~V}$ and $r_{D}=$ $0.0037 \Omega$. The filter inductors are IHLP-8787MZ from Vishay with $L=75 \mu \mathrm{H}$ and $r_{L F}=30 \mathrm{~m} \Omega$ at $25^{\circ} \mathrm{C}$. Taking into consideration the temperature effect, the value $r_{L F}=150 \mathrm{~m} \Omega$ is assumed. Upon substitution $V_{D}, r_{D}, V_{\text {Bat(Max) }}, r_{L F}$, and $I_{o}=25 \mathrm{~A}$ in (15), the efficiency of the rectifier stage at maximum charging current is $\eta_{R}=0.902$ then, the overall efficiency is $\eta=\eta_{I} \eta_{R}=0.863$. 
4) The characteristic impedance is obtained from (9), $Z_{p}=128 \Omega$. The parallel quality factor is calculated from (10), $Q_{p}=0.355$, which yields an initial value of the power factor angles $\phi_{1,2,3,4}=70^{\circ}$, well above of the minimum value $\phi_{z v s}=(0.7 \mu \mathrm{s} / 8 \mu \mathrm{s}) \cdot 360^{\circ}=31.5^{\circ}$. From Table I, the reactive components are: $L=Z_{p} / \omega_{p}=163 \mu \mathrm{H}$ and $C_{p}=4 / \omega_{p} Z_{p}=40 \mathrm{nF}$. The output capacitor is $C_{o}=220 \mu \mathrm{F}$.

\section{EXPERIMENTAL RESULTS}

The practical components of the resonant tank used in the prototype are: $L=160 \mu \mathrm{H}, C_{p}=40 \mathrm{nF}$. The capacitor $C_{s}$ is chosen high enough for blocking the DC current through the transformer, $C_{s}=1 \mu \mathrm{F}$. The practical implementation was carried out using the IRF840LC MOSFET transistor with the IR2111 circuit as the driver. The resonant inductors were implemented using the core RM12 of 3C96 material. As the number of phases is even $(N=4)$, the full control of the charging current is achieved by shifting the phases by pairs, i.e. $\Psi_{0}=\Psi_{1}=0^{\circ}$ and $\Psi_{2}=\Psi_{3}=\Psi$. Experimental waveforms are shown in Figs. 9 and 10.

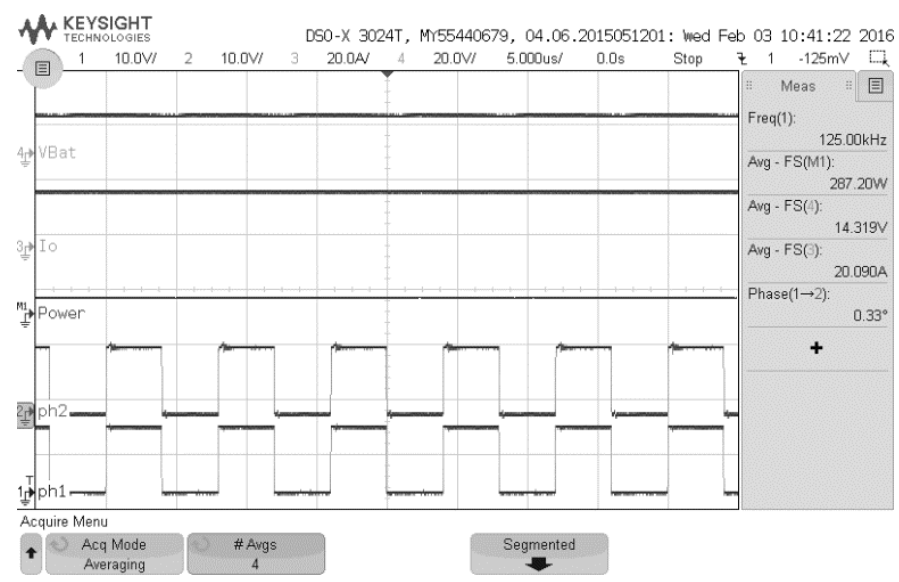

Fig. 9. Battery voltage, charge current and control signals at maximum charging rate, $I_{o}=20 \mathrm{~A}$.

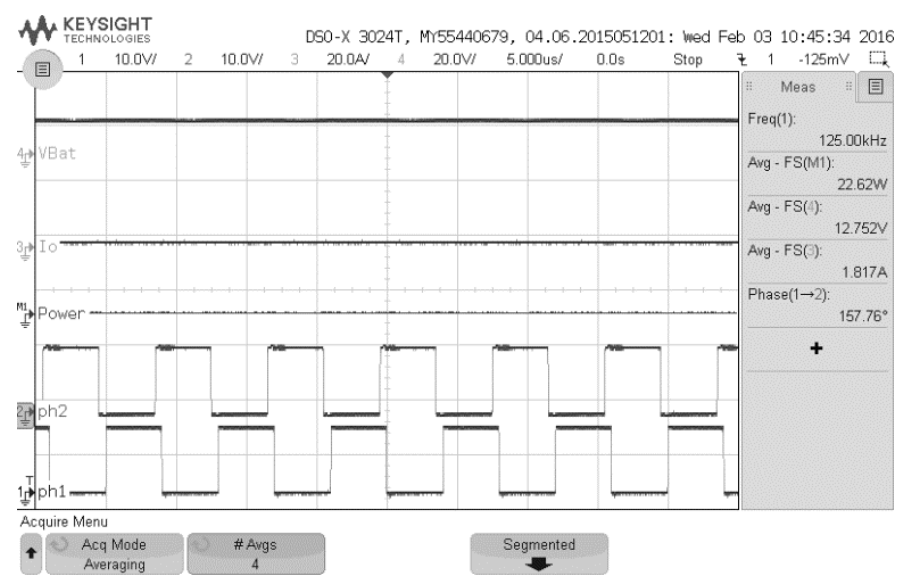

Fig. 10. Battery voltage, charge current and control signals at minimum charging rate, $I_{o}=1.8 \mathrm{~A}$.

The maximum value of experimental efficiency of the proposed charger was $\eta=84 \%$ and was achieved at $\Psi=101^{\circ}$. The experimental efficiency is lower than the theoretical value due to the transformer, PCB, connectors among others losses not included in the model.

\section{CONCLUSIONS}

The analysis and design of a multiphase resonant converter for battery charger applications has been presented. Since the load current in the AC side is shared among four equal sections, the circuit presents high output current capability using low cost power MOSFETs switching at high frequency and consequently requiring small magnetic components. The proposed output rectifier based on a current multiplier also diminishes the conduction losses by using passive components. The control is carried out at constant frequency, by adjusting the phase displacement of the drive signals, while maintaining the zero voltage switching (ZVS) mode at any operation point. The converter is designed to meet the entire requirement from the battery to extend its service life and preserve the state of health.

\section{ACKNOWLEDGEMENT}

This work is sponsored by the Spanish Ministry of Economy and Competitiveness, the Principality of Asturias Government and the European Union (ERFD), under Research Grants FC15-GRUPIN14-073 and DPI2013-46541-R and the Spanish Ministry of Science and the EU through the project TEC201452316-R: 'Estimation and Optimal Control for Energy Conversion with Digital Devices’ ECOTRENDD.

\section{REFERENCES}

[1] - Joern Albers, Eberhard Meissner and Sepehr Shirazi "Lead-acid batteries in micro-hybrid vehicles”, Journal of Power Sources 196, pages 39934002. 2011.

[2] - Xue Liu, C.A. Baguley, U.K. Madawala, D.J. Thrimawithana, “A Compact Power Converter for High Current and Low Voltage Applications”, Proceedings of the $39^{\text {th }}$ Annual Conference of the IEEE Industrial Electronics Society, IECON2013, pp. 140-144.

[3] - Ashok Bindra, "Wide-Bandgap-Based Power Devices", IEEE Power Electronics Magazine, Vol. 2, No. 1, pp. 42-47, March 2015.

[4] - Mariusz Bojarski, Erdem Asa, Kerim Colak, Dariusz Czarkowski, “A 25 kW Industrial Prototype Wireless Electric Vehicle Charger”, Proceedings of the 2016 IEEE Applied Power Electronics Conference and Exposition, APEC '2016. pp. 1756-1761.

[5] - C. Branas, F.J. Azcondo, R. Casanueva, "A Generalize Study of Multiphase Parallel Resonant Inverters for High-Power Applications”, Circuits and Systems I: Regular Papers, IEEE transactions on, Vol. 55, No. 7, pp. 2128-2138, Aug. 2008.

[6] - L. Huber, Milan M. Jovanovic, "Forward-flyback Converter with Current-Doubler Rectifier: Analysis, Design and Evaluation Results”, IEEE Trans. on Power Electronics, Vol. 14 No. 2, pp.184-192, Jan. 1999.

[7] - P. Alou, J. A. Oliver, O. García, R. Prieto, J. A. Cobos, "Comparison of Current Doubler Rectifier and Center Tapped Rectifier for Low Voltage Applications", Proceedings of the Twenty-First Annual IEEE Applied Power Electronics Conference and Exposition, 2006. APEC '06. pp. 744750 .

[8] - R. W. Erickson, D. Maksimovic, “Fundamentals of Power Electronics”, $2^{\text {nd }}$ Ed. Kluwer Academic Publishers, 2001.

[9]- D. Czarkowski, M.K. Kazimierczuk, "Phase-Controlled Series-Parallel Resonant Converter," IEEE Trans. on Power Elect., Vol.8, No.3, July 1993. pp. 309-319.

[10] - Lopez, V.M.; Navarro-Crespin, A.; Schnell, R. W.; Branas, C.; Azcondo, F. J.; Zane, R. "Current Phase Surveillance in Resonant Converters for Electric Discharge Applications to Assure Operation in Zero-VoltageSwitching Mode”, Power Electronics, IEEE Trans. on, Vol. 27 , 2012 , pp. $2925-2935$. 